14

\title{
Имитация синаптической связи нейроноподобных генераторов с помощью мемристивного устройства
}

\author{
() С.А. Герасимова, А.Н. Михайлов, А.И. Белов, Д.С. Королев, О.Н. Горшков, В.Б. Казанцев \\ Национальный исследовательский Нижегородский государственный университет им. Н.И. Лобачевского, \\ 603950 Нижний Новгород, Россия \\ e-mail: gerasimova@neuro.nnov.ru
}

(Поступило в Редакцию 14 сентября 2016 г.)

\begin{abstract}
Представлена физическая модель синаптически связанных нейроноподобных генераторов, взаимодействующих с помощью мемристивного устройства. Модель имитирует синаптическую передачу импульсных сигналов между нейронами мозга. Воздействие на принимающий генератор осуществляется через мемристивное устройство, демонстрирующее адаптивное поведение. Установлено, что предложенный канал связи обеспечивает вынужденную синхронизацию, параметры которой зависят от чувствительности мемристивного устройства. Экспериментально получены режимы синхронизации с соотношением частот $1: 1,2: 1$.
\end{abstract}

DOI: 10.21883/JTF.2017.08.44735.2033

\section{Введение}

Разработка перспективных нейроморфных компактных устройств (в том числе микро- и наночипов), воспроизводящих информационно-вычислительные функции мозга, является в настоящее время одним из прорывных направлений современной науки и техники. Это связано, прежде всего, с перспективными медицинскими приложениями - созданием искусственных нейрочиповимплантов, позволяющих осуществлять воздействие на электровозбудимые клетки (нервные клетки - нейроны, мышечные клетки сердца - кардиомиоциты), управлять их активностью, а также замещать поврежденную нервную ткань. Другим актуальным направлением является приложение таких устройств к решению актуальных задач в сфере информационно- телекоммуникационных технологий - созданию интеллектуальных мозгоподобных систем автоматического управления и синхронизации, систем распознавания, кодирования и декодирования информации. Своевременность решения данных задач обусловлена, с одной стороны, успехами современной нейронауки, позволяющей получать данные о процессах, происходящих на уровне молекулярно-клеточной организации систем мозга, и, с другой стороны, развитием радиотехники, физики микро- и наноструктур, твердотельной наноэлектроники.

Одним из направлений, определяющих развитие нейроморфных систем, является исследование возможности создания простых электронных аналогов нейрона и синапса с использованием тонкопленочных мемристивных наноструктур и устройств на их основе [1]. Мемристивное устройство представляет собой физическую модель мемристора - элемента электрических цепей, способного менять сопротивление в зависимости от величины поступающего на вход электрического сигнала. Способность мемристивной структуры менять проводимость под воздействием импульсных сигналов делает ее почти идеальным электронным аналогом си- напсов (соединений между нейронами). Более сложный подход к исследованию нейроморфных систем, основанных на мемристивных устройствах, заключается в использовании таких устройств для реализации правил обучения на основе синаптической пластичности (возможности адаптивного изменения силы связи между нейронами в зависимости от активности). Правило пластичности, сформулированное Хэббом, утверждает, что, когда один нейрон активирует другой нейрон, связь между нейронами усиливается. Зависимость эффективности синаптической связи (усиление связи потенциация, спад - депрессия) от соотношения времен прихода импульса на синапс (механизм spike timing dependent plasticity - STDP) была продемонстрирована в мемристивных системах (см., например, обзоры [2,3]). Для демонстрации различных форм STDP-механизма используют непрерывный (аналоговый) характер изменения сопротивления в мемристивных структурах под действием пре- и постсинаптических сигналов с формой, подобной биологическим импульсам - спайкам [4]. Для генерации таких сигналов используются специальные электрические схемы на основе стандартных цифровых элементов. В то же время известно, что для генерации спайкоподобной активности могут быть использованы как сами мемристивные устройства [5], так и простые схемы на их основе [6]. Это позволяет рассчитывать в ближайшей перспективе на полный отказ от гибридного аналого-цифрового подхода для построения нейросетевых архитектур на основе мемристивных устройств.

Независимо от того, какое модельное устройство будет использоваться в качестве нейрона, ключевой задачей является разработка и реализация сложных нейроморфных систем на основе взаимодействующих нейроноподобных элементов. Для воспроизведения нейроноподобного сигнала можно использовать упрощенные электрические схемы, качественно характеризующие нейрональную активность - возможность переключения между возбудимым и колебательным режимами 
(электрическая модель ФитцХью-Нагумо [7], Дмитриева [8], Некоркина [9], Щапина [10]). Существующие теоретические работы показывают, что мемристивное устройство способно обеспечить адаптивную связь (синхронизацию) между нелинейными системами, представленными, например, цепочками Чуа [11]. Однако экспериментально такое взаимодействие на примере двух генераторов через реальные мемристивные устройства практически не исследовано.

В настоящей работе изучена стохастическая природа изменения резистивного состояния мемристивного устройства при воздействии периодических электрических сигналов разной формы. Впервые экспериментально показано, что изменение сопротивления мемристивных устройств под действием спайкоподобных сигналов обеспечивает адаптивную связь и синхронизацию нейроноподобных генераторов.

\section{Параметры мемристивных устройств}

В качестве мемристивных устройств использованы тонкопленочные наноструктуры ,металл-оксидметалл“" на основе различных оксидных диэлектриков: $\mathrm{Au} / \mathrm{SiO}_{2} / \mathrm{TiN} / \mathrm{Ti}$ и $\mathrm{Au} / \mathrm{ZrO}_{2}(\mathrm{Y}) / \mathrm{TiN} / \mathrm{Ti}$. Структуры были получены с помощью метода магнетронного распыления на окисленных подложках кремния. Толщина рабочего диэлектрика $\left(\mathrm{SiO}_{2}\right.$ или $\left.\mathrm{ZrO}_{2}(\mathrm{Y})\right)$ составляла $40 \mathrm{~nm}$, толщина верхнего электрода $\mathrm{Au} 40 \mathrm{~nm}$, толщины составных слоев нижнего электрода TiN и Ti по $25 \mathrm{~nm}$. Детали технологических операций представлены в работах $[12,13]$.

Вольт-амперные характеристики структур, демонстрирующие воспроизводимое переключение между состоянием с низким сопротивлением (low-resistance state LRS) и состоянием с высоким сопротивлением (highresistance state - HRS), показаны на рис. $1[11,12]$. Резистивное переключение обусловлено окислением и восстановлением участков проводящих каналов (филаментов) в оксидной пленке при приложении напряжения разной полярности. Соответствующие изменения тока обозначены на рис. 1 как RESET и SET. Степень резистивного переключения (изменения величины сопротивления структур) зависит от параметров электрического воздействия и определяет адаптивное (синаптическое) поведение мемристивных наноструктур [13].

Видно, что для обоих видов наноструктур резистивное переключение характеризуется определенным разбросом значений напряжений переключения, а также значений сопротивлений (токов) в состояниях LRS и HRS, что связано со стохастической природой микроскопических процессов разрушения и восстановления филаментов. Несмотря на общее качественное поведение, есть и ряд различий в параметрах резистивного переключения, которые связаны с характеристиками используемых оксидных пленок. В частности, для полного завершения процесса SET, который соответствует уменьшению сопротивления мемристивной структуры, в случае
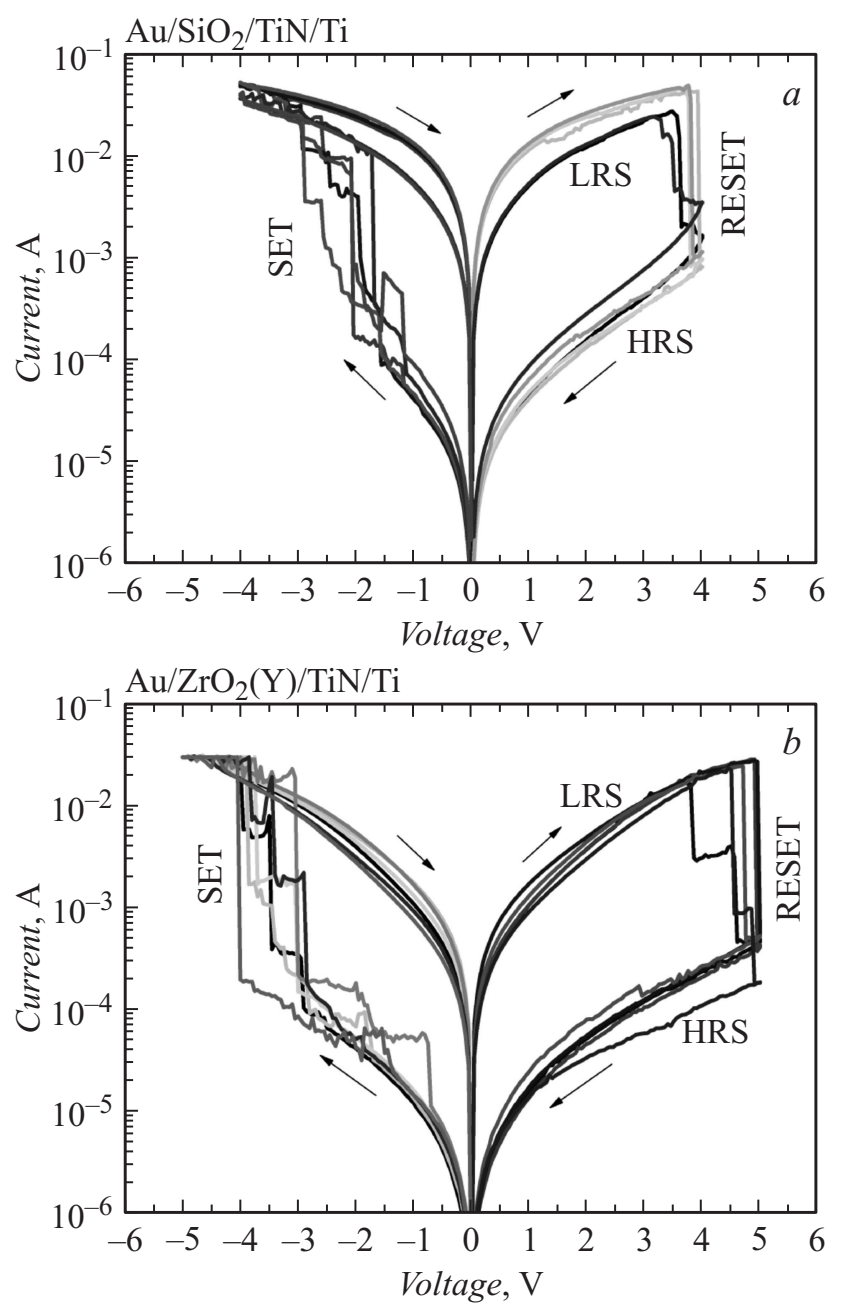

Рис. 1. Вольт-амперные характеристики мемристивных наноструктур на основе $\mathrm{SiO}_{2}(a)$ и $\mathrm{ZrO}_{2}(\mathrm{Y})(b)$.

$\mathrm{Au} / \mathrm{SiO}_{2} / \mathrm{TiN} / \mathrm{Ti}$ достаточно отрицательного напряжения в диапазоне $1.5-3 \mathrm{~V}$, в то время как для $\mathrm{Au} / \mathrm{ZrO}_{2}(\mathrm{Y}) / \mathrm{TiN} / \mathrm{Ti}$ это напряжение находится в диапазоне $3-4.5 \mathrm{~V}$. Следует отметить, что для реализации RESET требуется большее напряжение, чем для SET. Как будет видно далее, такая асимметрия важна для понимания отклика мемристивной структуры на биполярные спайкоподобные сигналы.

\section{Адаптивное поведение мемристивных устройств}

В ходе предварительных экспериментов было проведено качественное исследование влияния сигналов различной формы (прямоугольный импульс отрицательной и положительной полярностей, „сложный“ сигнал кардиоритм), амплитуды и частоты на возможность переключения мемристивного устройства. Схема соединения мемристивного устройства и генератора показана на рис. 2. Сигнал с выхода генератора поступает на верхний электрод (вход) мемристивной структуры, с 


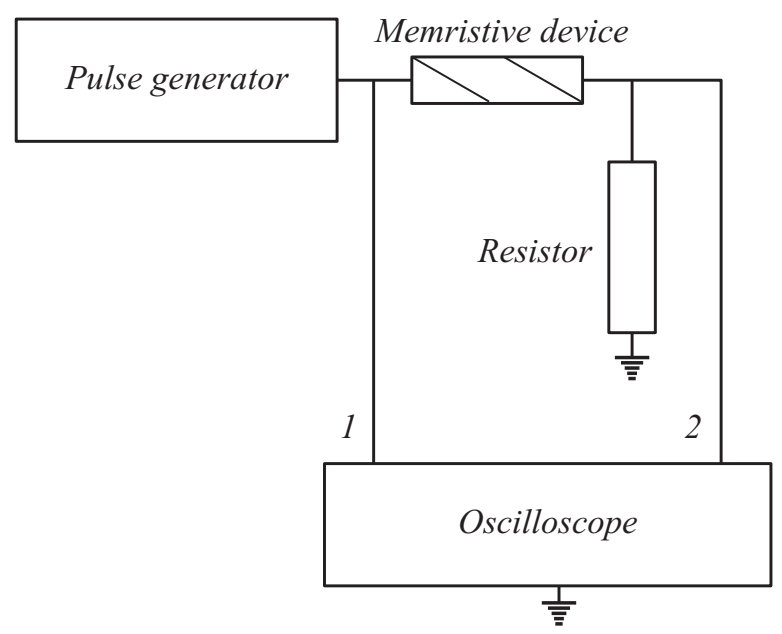

Рис. 2. Блок-схема соединения мемристивного устройства и генератора произвольных сигналов.
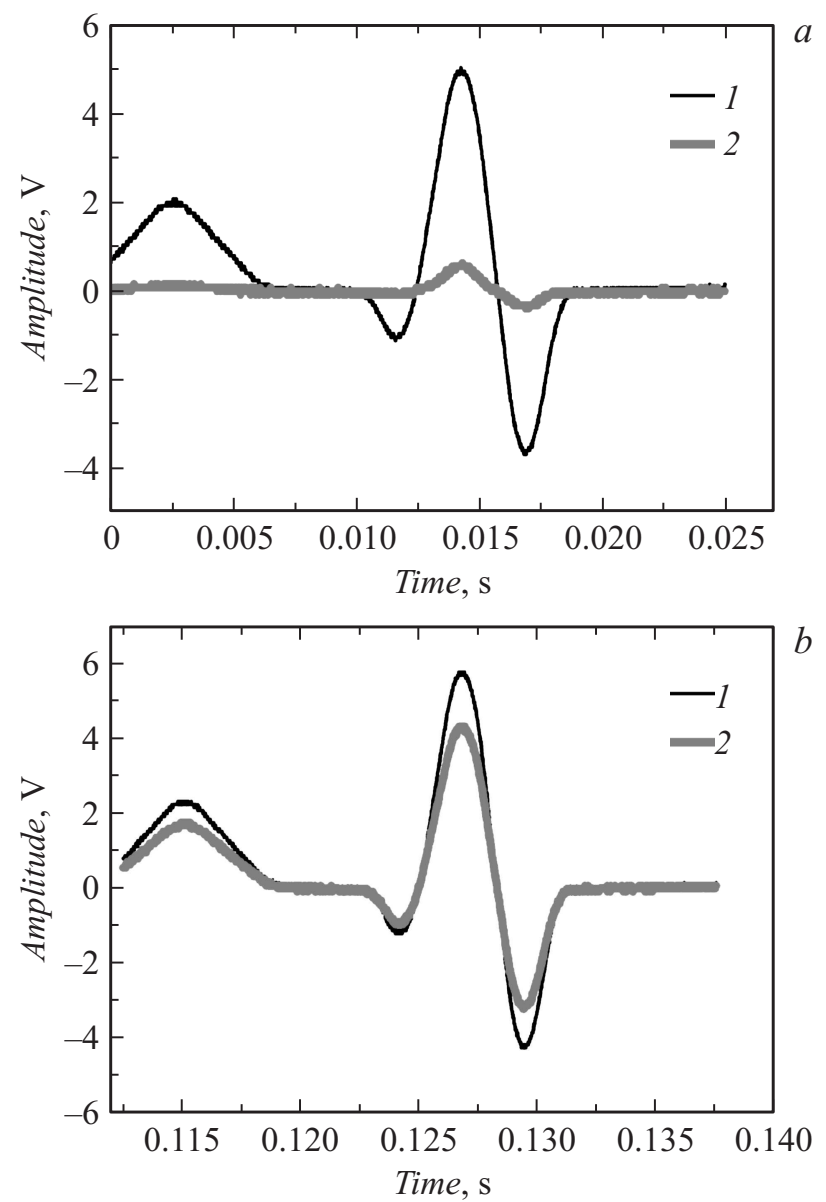

Рис. 3. Результаты экспериментов по переключению мемристивной структуры $\mathrm{Au} / \mathrm{SiO}_{2} / \mathrm{TiN} / \mathrm{Ti}$ из более проводящего состояния в менее проводящее состояние $(a)$ и обратно $(b)$ при воздействии кардиоритма.

нижнего электрода (выхода) мемристивной структуры сигнал идет на второй канал осциллографа через нагрузочное сопротивление. С выхода генератора сигнал фик- сируется на первом канале осциллографа. Исследования проводились с помощью генератора WaveStation 2012.

Показано, что форма сигнала влияет на способность переключения модели мемристора из высокоомного состояния в низкоомное и обратно. Для структуры $\mathrm{Au} / \mathrm{SiO}_{2} / \mathrm{TiN} / \mathrm{Ti}$ были зафиксированы переходы из высокоомного $(5 \mathrm{k} \Omega)$ в низкоомное $(100 \Omega)$ состояние с увеличением амплитуды воздействующего сигнала от 1 до $5 \mathrm{~V}$ при разной форме воздействующего сигнала генератора.

На рис. 3 представлены результаты эксперимента по переключению мемристивной структуры $\mathrm{Au} / \mathrm{SiO}_{2} / \mathrm{TiN} / \mathrm{Ti}$ из более проводящего состояния в менее проводящее и обратно. Переход осуществлен под действием сигнала, моделирующего кардиоритм. На рис. 3 кривая 1 представляет сигнал с выхода генератора до воздействия на модель мемристора (точка 1 на схеме рис. 2), кривая 2 - сигнал с выхода мемристивного устройства при нагрузочном сопротивлении $1 \mathrm{k} \Omega$ (точка 2 на схеме рис. 2). Сопротивление мемристивной структуры изменялось от $5 \mathrm{k} \Omega$ (при амплитуде сигнала генератора от 1 до $5 \mathrm{~V}$ ) до $100 \Omega$ при увеличении амплитуды сигнала генератора до 6-7V. Изменение сигнала в точке 1 характеризует изменение сопротивления мемристивной структуры. При переходе мемристивного устройства в более проводящее состояние сигналы становятся сравнимы по амплитуде, поскольку подавляющая часть напряжения начинает падать на нагрузочном сопротивлении (рис. $3, b$ ). При смене полярности сигнала мемристор переходил в менее проводящее состояние, в таком случае сигналы в точках 1 и 2 отличались более чем в 5 раз (рис. 3,a). Стоит отметить, что при амплитуде воздействующего сигнала (рис. 2, точка 1) около $6 \mathrm{~V}$ происходило самопроизвольное изменение состояния мемристивной структуры $\mathrm{Au} / \mathrm{SiO}_{2} / \mathrm{TiN} / \mathrm{Ti}$, по-видимому, обусловленное изменением структуры филамента и его проводящих свойств.

Таким образом, результаты предварительных экспериментов с применением сигналов различной формы свидетельствуют об адаптивном поведении мемристивного устройства, поэтому можно считать, что соединение нейроноподобных генераторов на основе мемристора будет имитировать свойство пластичности.

Для построения системы связанных нейроноподобных генераторов через мемристивное устройство было проведено исследование характеристик переключения мемристивного образца из высокоомного в низкоомное состояние под воздействием радиотехнического нейроноподобного генератора (в той же схеме, что представлена на рис. 2). Были проведены эксперименты по изучению влияния импульсного нейроноподобного сигнала различной амплитуды и частоты следования импульсов на возможность переключения мемристора из более проводящего состояния в менее проводящее. Было выявлено, что частота использованного нейроноподобного сигнала в исследованном диапазоне $2-150 \mathrm{~Hz}$ не влияет на возможность переключения мемристора. 
Имитация нейронной активности в настоящей работе осуществлялась с помощью радиотехнического генератора, аналогичного генератору ФитцХью-Нагумо [7]. Электрическая схема нейроноподобного генератора разрабатывалась в программе MicroCap.

Динамика выходного сигнала генератора ФитцХьюНагумо качественно отражает основные свойства нервного импульса - спайка. С помощью потенциометра реализована возможность переключения между автоколебательным и возбудимым динамическими режимами. В схеме реализована возможность изменения характеристик колебательного контура (частота, длительность импульсов). Кубическая нелинейность реализована двумя ветками диодов. Амплитуда выходного сигнала увеличивается с помощью операционного усилителя mc1458 от 0.6 до $5 \mathrm{~V}$, возможность варьирования амплитуды осуществлена с помощью потенциометра.

Воздействие нейроноподобного генератора на мемристивную структуру $\mathrm{Au} / \mathrm{SiO}_{2} / \mathrm{TiN} / \mathrm{Ti}$ изучалось путем измерения сопротивления мемристивной структуры и регистрации сигналов в точках 1 и 2 схемы, представленной на рис. 2 , в зависимости от амплитуды сигнала на входе мемристивной структуры.

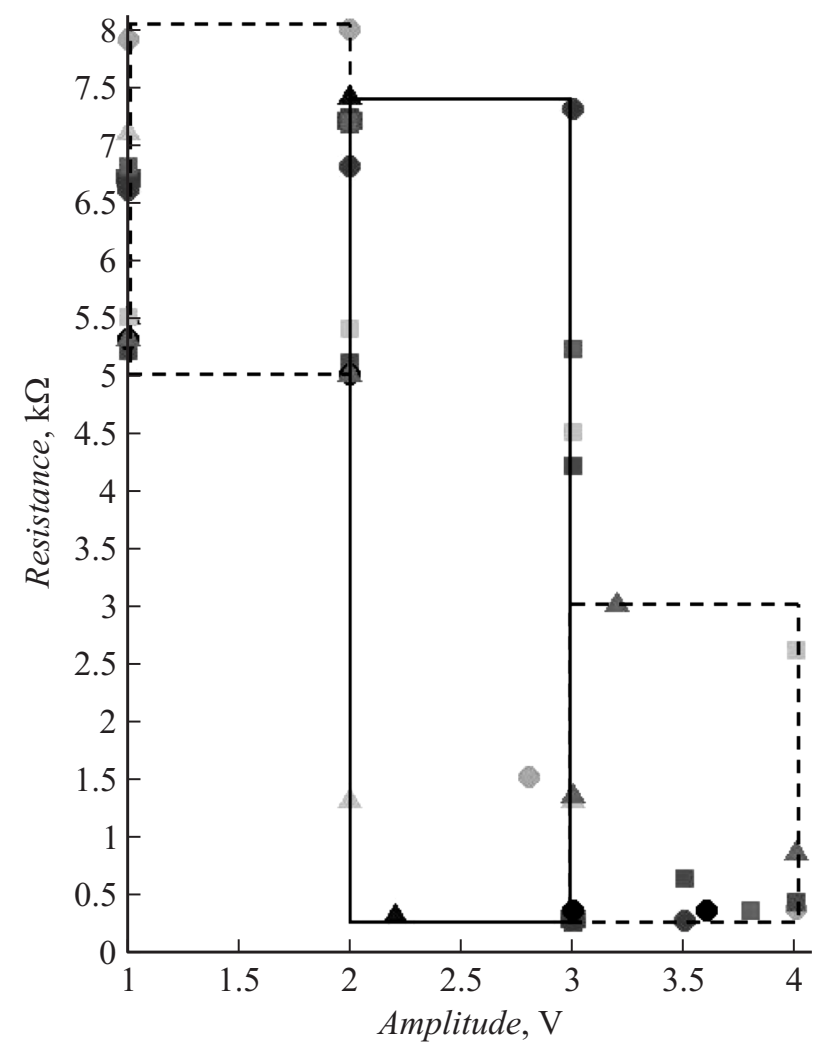

Рис. 4. Зависимость сопротивления мемристивного устройства $\mathrm{Au} / \mathrm{SiO}_{2} / \mathrm{TiN} / \mathrm{Ti}$ от амплитуды сигнала нейроноподобного генератора. Прямоугольниками показаны диапазоны значений, соответствующие разбросу сопротивлений в состояниях до и после переключения, а также в процессе переключения мемристивного устройства.

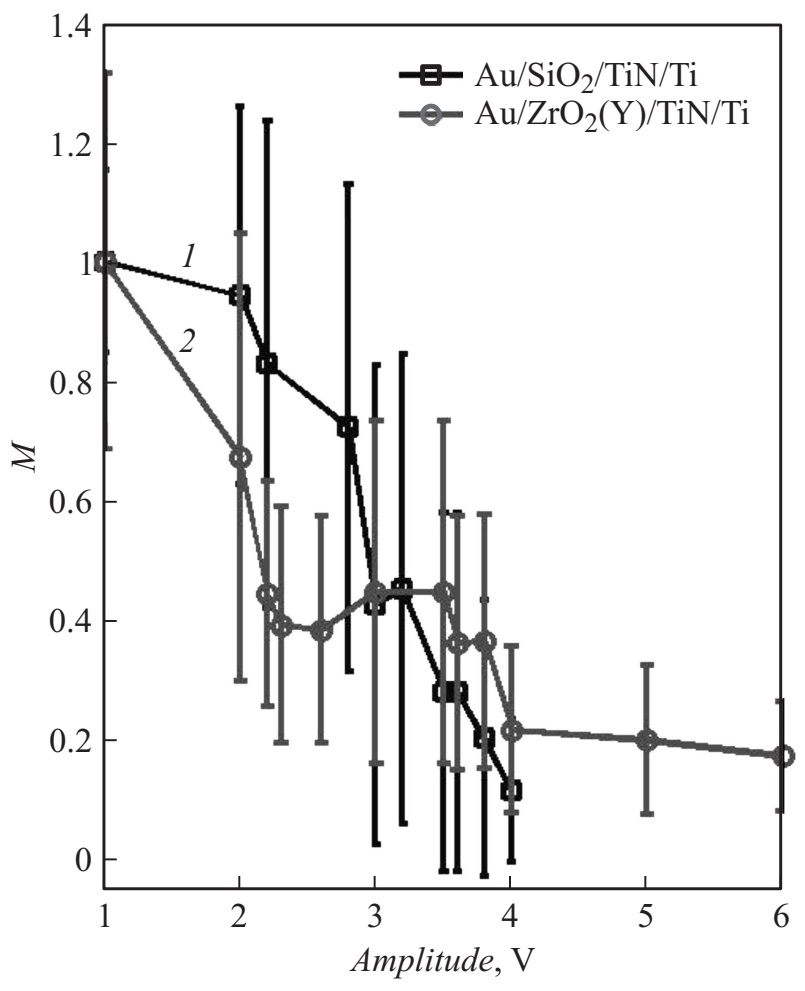

Рис. 5. Зависимости среднего относительного изменения сопротивления мемристивных наноструктур $\mathrm{Au} / \mathrm{SiO}_{2} / \mathrm{TiN} / \mathrm{Ti}$ и $\mathrm{Au} / \mathrm{ZrO}_{2}(\mathrm{Y}) / \mathrm{TiN} / \mathrm{Ti}$ от амплитуды сигнала воздействующего нейроноподобного генератора.

Из рис. 4 следует, что исследуемый образец переключается при воздействии сигнала нейроноподобного генератора в более проводящее состояние при амплитуде от 2 до $3 \mathrm{~V}$. При этом следует отметить большой разброс значений сопротивления в начальном и конечном состояниях, что связано с отмеченной выше стохастической природой резистивного переключения [1]. Также имеет место большой разброс значений амплитуды нейроноподобного сигнала (точка 2 на схеме рис. 2), при воздействии которого происходит изменение резистивного состояния.

В диапазоне амплитуд воздействия $2-3 \mathrm{~V}$, в котором реализуется реакция на внешнее воздействие (рис. 4), были зафиксированы самопроизвольные изменения состояния мемристивной структуры, что также характеризует стохастическую природу резистивного переключения.

Для сравнения реакции разных образцов мемристивных структур $\mathrm{Au} / \mathrm{SiO}_{2} / \mathrm{TiN} / \mathrm{Ti}$ и $\mathrm{Au} / \mathrm{ZrO}_{2}(\mathrm{Y}) / \mathrm{TiN} / \mathrm{Ti}$, характеризующихся разными параметрами переключения их из высокоомного в низкоомное состояние, была использована нормировка значений сопротивления мемристивного элемента на значение сопротивления в исходном состоянии $R_{1}$. Относительное изменение сопротивления вычислялось по формуле

$$
\delta R=R / R_{1}
$$


Были вычислены средние значения относительного изменения сопротивления мемристивной структуры $M=\overline{\delta R}$ и стандартные отклонения $S$ :

$$
\begin{gathered}
M=\overline{\delta R}=\frac{1}{n} \sum_{i=1}^{n} \delta R_{i} \\
S=\left(\frac{1}{n-1} \sum_{i=1}^{n}\left(\delta R_{i}-\overline{\delta R}\right)^{2}\right)^{1 / 2}
\end{gathered}
$$

где $\delta R_{i}-i$-й элемент выборки, $n-$ объем выборки по значению сопротивления мемристивной структуры $R$.

На рис. 5 круглыми и квадратными точками обозначены средние значения относительного изменения сопротивления мемристивного элемента $M$ от амплитуды воздействия сигнала нейроноподобного генератора с учетом вычисленных стандартных отклонений для образцов $\mathrm{Au} / \mathrm{SiO}_{2} / \mathrm{TiN} / \mathrm{Ti}$ и $\mathrm{Au} / \mathrm{ZrO}_{2}(\mathrm{Y}) / \mathrm{TiN} / \mathrm{Ti}$ (кривая 1 и кривая 2 соответственно). Видно, что в определенном диапазоне амплитуд входного сигнала происходит переключение мемристивного устройства в более проводящее состояние. Для образца $\mathrm{Au} / \mathrm{SiO}_{2} / \mathrm{TiN} / \mathrm{Ti}$ (кривая 1) характерны изменения сопротивления от $8.5 \mathrm{k} \Omega$ до $140 \Omega$ при амплитуде воздействия нейроноподобного генератора около $4 \mathrm{~V}$. Сопротивление структуры $\mathrm{Au} / \mathrm{ZrO}_{2}(\mathrm{Y}) / \mathrm{TiN} / \mathrm{Ti}$ (кривая 2) изменяется от $30 \mathrm{k} \Omega$ до $150 \Omega$ при амплитуде входного сигнала до $6 \mathrm{~V}$, что согласуется с бо́льшими значениями напряжения резистивного пере-

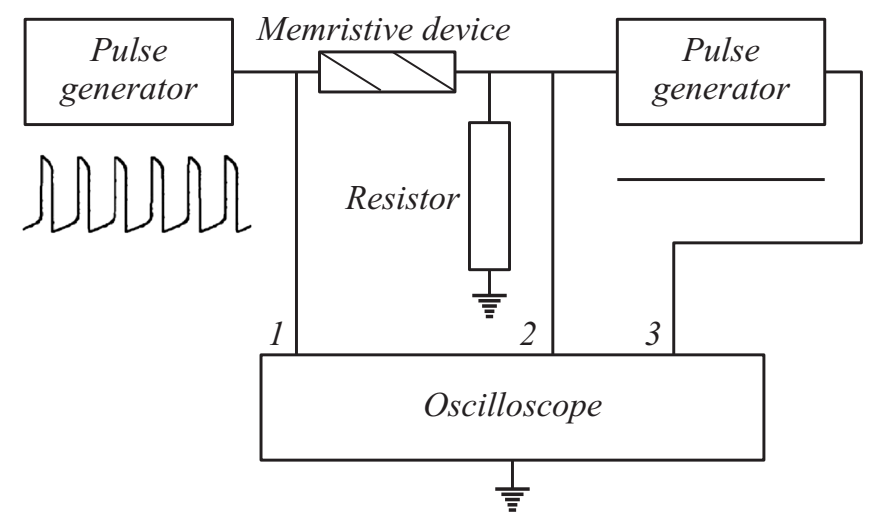

No coupling
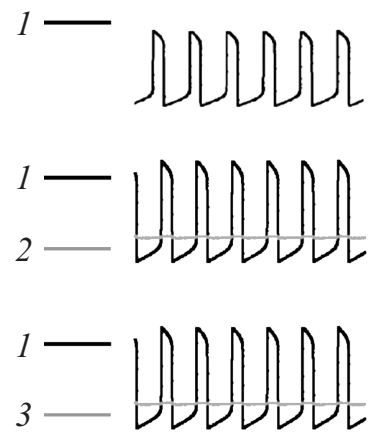

Рис. 6. Схема эксперимента по взаимодействию нейроноподобных генераторов с помощью мемристивного устройства.
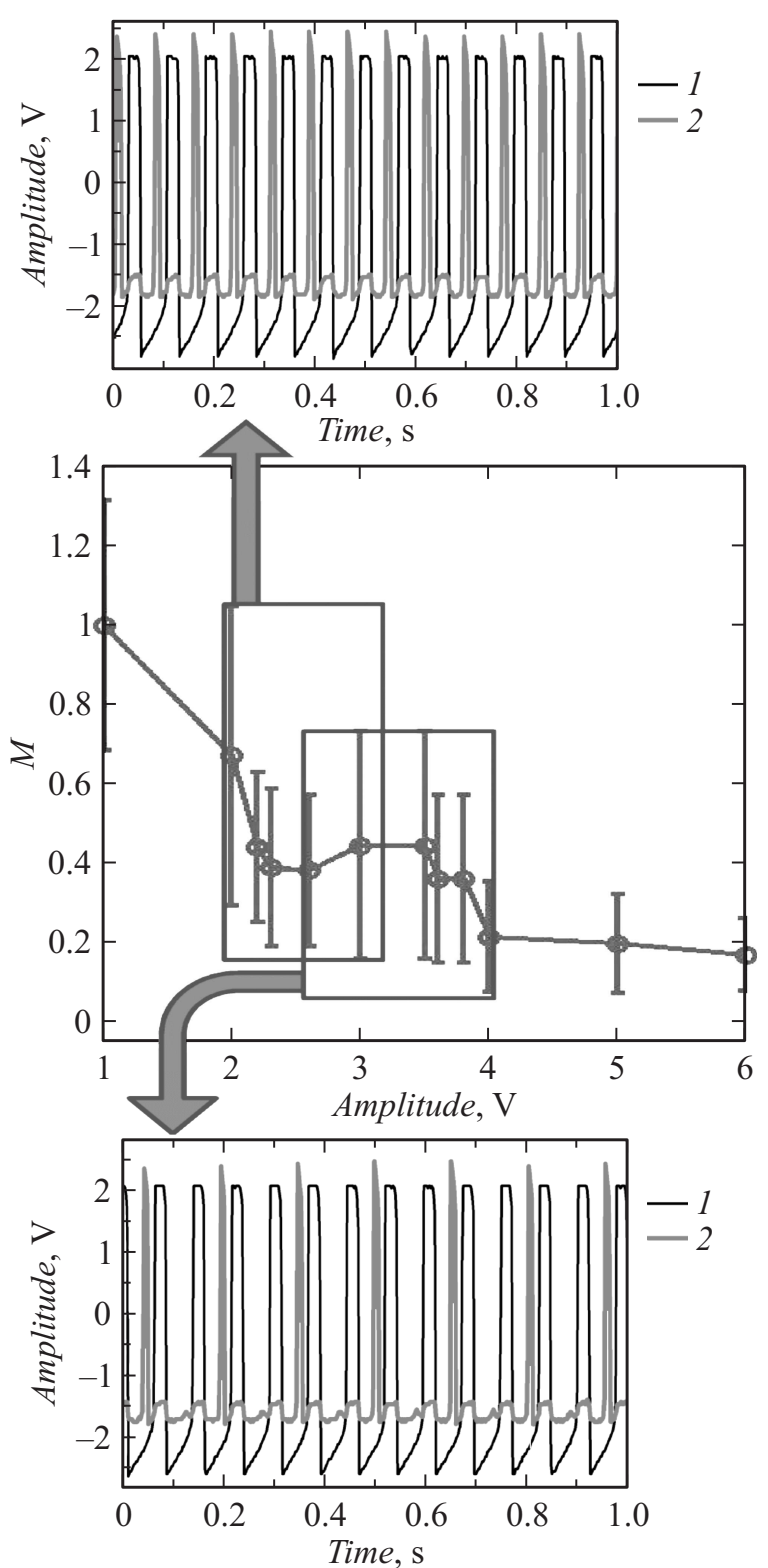

Рис. 7. Результаты экспериментов по взаимодействию нейроноподобных генераторов с помощью мемристивного устройства.

ключения при измерении вольт-амперных характеристик для данного образца (рис. 1).

\section{Синаптическое соединение нейроподобных генераторов}

После настройки и отладки управляющего и управляемого нейроноподобных генераторов ФитцХью-Нагумо был проведен эксперимент по исследованию взаимодействия генераторов с использованием мемристивной структуры в качестве синаптического соединения. Схема эксперимента представлена на рис. 6. 

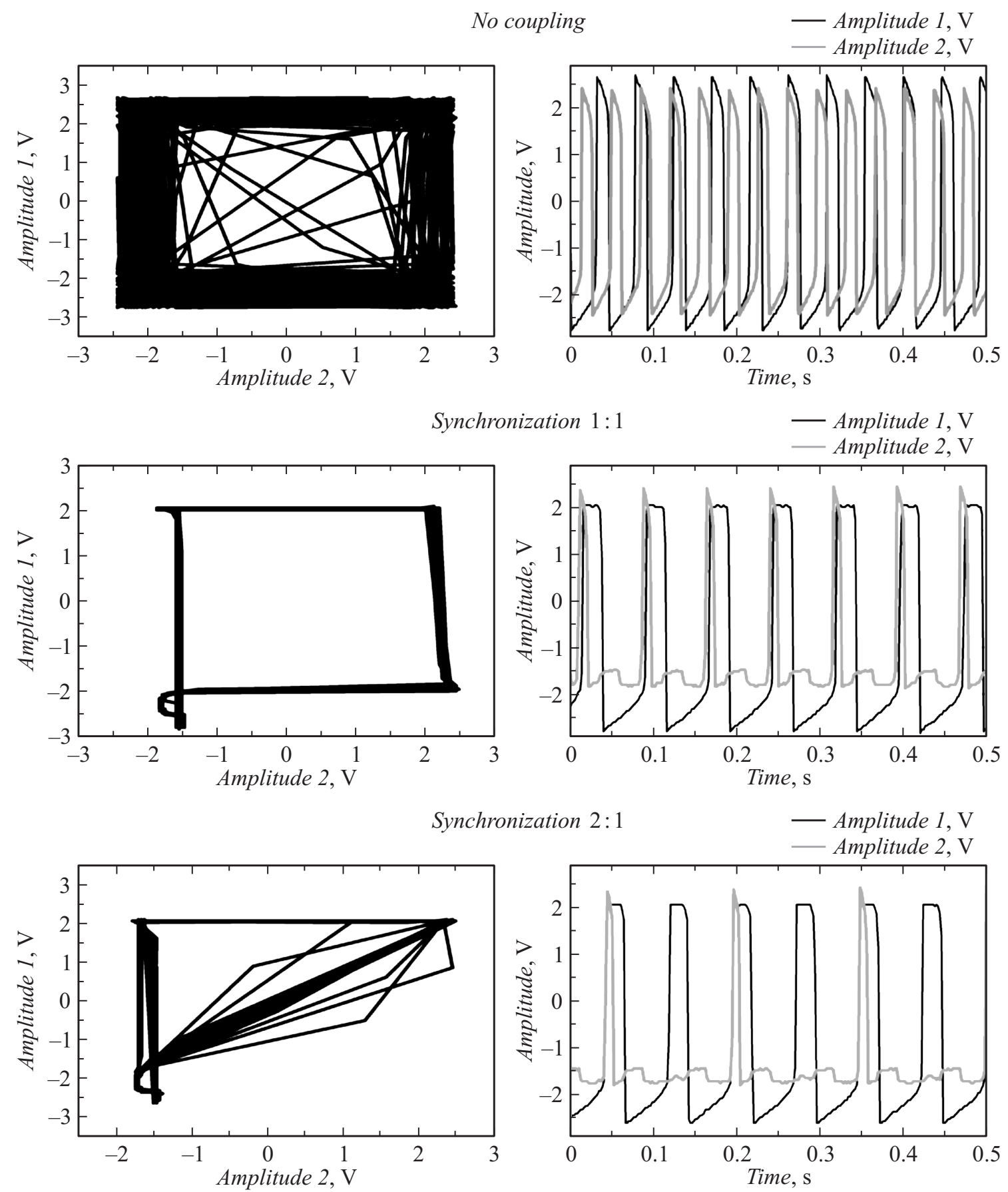

Рис. 8. Амплитуды сигналов нейроноподобных генераторов, связанных через мемристивное устройство, при отсутствии синхронизации и в случаях синхронизации $1: 1,2: 1$ (плоскость амплитуд взаимодействующих генераторов).

Эксперимент проводился следующим образом. В начальный момент времени управляющий генератор находился в автоколебательном режиме, управляемый генератор - в возбудимом режиме, в качестве синапса использовалась мемристивная структура $\mathrm{Au} / \mathrm{ZrO}_{2}(\mathrm{Y}) / \mathrm{TiN} / \mathrm{Ti}$, проявляющая более плавное изменение сопротивления под воздействием управляющего генератора - более широкий диапазон чувствительности к воздействию (рис. 5, кривая 2). Сигнал с управляющего генератора (точка 1 на рис. 6) поступал на вход мемристивного устройства, с выхода мемристивного устройства (точка 2 на рис. 6) приходил на вход управляемого генератора через нагрузочное сопротивление. Сигналы в точках 1 и 2,1 и 3 на схеме фиксировались осциллографом (точка 3 - выход управляемого генератора). При увеличении амплитуды сигнала управляющего генератора регистрировалось воздействие на модель мемристора (путем сравнения 
сигналов в точках 1 и 2 на схеме). При переходе мемристивного устройства из высокоомного состояния в более низкоомное состояние происходило установление связи между двумя нейроноподобными генераторами. Управляемый генератор переходил в колебательный режим, при этом сигналы генераторов синхронизовались. При дальнейшем увеличении амплитуды управляющего нейроноподобного генератора были зафиксированы режимы синхронизации $1: 1,1: 2$. Параметром, характеризующим силу связи, являлось сопротивление мемристивной структуры, причем для наблюдения различных режимов синхронизации необходимо, чтобы сопротивление мемристивной структуры менялось плавно в большом диапазоне амплитуд управляющего генератора. Так, для структуры $\mathrm{Au} / \mathrm{ZrO}_{2}(\mathrm{Y}) / \mathrm{TiN} / \mathrm{Ti}$, удовлетворяющей этому требованию, различные режимы синхронизации нейроноподобных генераторов наблюдаются при амплитуде сигнала управляющего генератора в диапазоне 2-3 V и сопротивлении мемристивной структуры в диапазоне $5-7 \mathrm{k} \Omega$ (рис. 7).

Для подтверждения эффекта синхронизации были построены графики зависимости амплитуды сигнала пресинаптического и постсинаптического электронного нейрона, а также осциллограммы (рис. 8). Видно, что при отсутствии синхронизации на плоскости амплитуд (рис. 8) не наблюдается замкнутой траектории, а временна́я зависимость показывает, что колебания асинхронны. При установлении связи между двумя генераторами в режиме синхронизации $1: 1$ возникает замкнутая траектория. При наблюдении режима синхронизации $2: 1$ основные точки сосредоточены вдоль главной диагонали, что указывает на наличие фазовой синхронизации импульсных сигналов. Точки, находящиеся справа от главной диагонали, соответствуют недостаточной частоте дискретизации сигнала на осциллографе на больших временных отрезках до $10 \mathrm{~s}$. Наличие петли слева от главной диагонали соответствует дробной синхронизации $2: 1$.

\section{Заключение}

В настоящей работе экспериментально продемонстрировано адаптивное поведение мемристивных наноструктур $\mathrm{Au} / \mathrm{SiO}_{2} / \mathrm{TiN} / \mathrm{Ti}$ и $\mathrm{Au} / \mathrm{ZrO}_{2}(\mathrm{Y}) / \mathrm{TiN} / \mathrm{Ti}$, проявляющих воспроизводимое биполярное резистивное переключение под воздействием сигналов различной формы, в том числе сигнала нейроноподобного генератора ФитцХьюНагумо. Экспериментально реализована схема синаптической связи нейроноподобных генераторов с помощью мемристивного устройства, найдены характеристики такой связи. Для случая мемристивного устройства $\mathrm{Au} / \mathrm{ZrO}_{2}(\mathrm{Y}) / \mathrm{TiN} / \mathrm{Ti}$ с более широким диапазоном чувствительности к воздействию входного сигнала показаны различные режимы модуляции сигнала: синхронизации $1: 1,2: 1$. Проведенное исследование содержит ключевые данные для построения нейроморфных моделей, ими- тирующих правила обучения нейронных сетей мозга, а также для разработки и реализации нейрогибридных систем, состоящих из управляющего устройства, мемристивной структуры и живой нейронной сети.

Работа выполнена при поддержке Российского научного фонда (грант № 16-19-00144).

\section{Список литературы}

[1] Adamatzky A., Chua L. Memristor Networks. 2013. 716 p.

[2] Kuzum D. et al. // Nanotechnology. IOP Publishing. 2013. Vol. 24. N 38. P. 382001.

[3] Thomas A. et al. // J. Phys. D. Appl. Phys. IOP Publishing. 2013. Vol. 46. N 9. P. 093001.

[4] Matveyev Y. et al. // J. Appl. Phys. AIP Publishing. 2015. Vol. 117. N 4. P. 044901.

[5] Mehonic A., Kenyon A.J. // Front. Neurosci. 2016. Vol. 10. N FEB. P. 0-10.

[6] Pickett M.D., Medeiros-Ribeiro G., Williams R.S. // Nature Materials. 2012. Vol. 12. P. 114-117.

[7] Герасимова C.A. и др. // Радиотехника и электроника. 2015. Т. 60. Вып. 8. С. 900-903.

[8] Дмитриев А.С. // Радиотехника и электроника. 2010. Т. 55. Вып. 4. С. 459-464.

[9] Binczak S. et al. // Neural Networks. 2006. Vol. 19. P. 684 693.

[10] Щапин Д.С. // Радиотехника и электроника. 2009. Т. 54. Вып. 2. С. 185-195.

[11] Frasca M. et al. // Phys. Stat. Sol. C. 2015. Vol. 12. N 1-2. P. 206-210.

[12] Gorshkov O.N. et al. // Письма в ЖТФ 2014. Т. 40. Вып. 2. C. 101-103.

[13] Mikhaylov A.N. et al. // Mater. Sci. Eng. B. Sol. Stat. Mater. Adv. Technol. 2015. Vol. 194. P. 48-54. 